\section{Toceranib toxicity in cats with neoplasia}

\section{Aaron Harper, Laura Blackwood}

University of Liverpool, Liverpool, UK

\section{AIM}

To retrospectively analyse treatment data of cats treated with toceranib to document adverse events related to the drug.

\section{METHODS}

The clinical records of the authors' institution were reviewed between 2009 and 2014. Cats were included in the study if they had received toceranib phosphate for at least two weeks and had at least one set of monitoring bloods (haematology, biochemistry) performed. Adverse events were categorised by the VCOG-CTAE grading system where appropriate. Cats with significant alterations in haematology and/or biochemistry or significant clinical signs before starting treatment with toceranib were excluded.

\section{RESULTS}

Eleven cats met the inclusion criteria. Median duration of treatment was 95 days (range 14-570 days). 7/11 cats had metastatic mast cell disease, the remaining 4/11 had carcinoma of various locations. All of the cats had gross disease and had received prior treatment with various combinations of surgery, radiotherapy and chemotherapy. VCOG grade I neutropenia occurred in 4/11 cats, and grade I lymphopenia occurred in one cat. Mild elevations in urea and creatinine each occurred in one cat and resolved after short treatment delays. One cat had marked elevations in ALT (>300 U/L) which did not resolve following cessation of treatment, although no clinical signs were observed. 5/11 cats demonstrated some gastro-intestinal side effects, mainly VCOG grade 1 vomiting, diarrhoea and/or inappetance. 7/11 cats died from disease progression. 2/11 died from heart disease, and it is unknown whether this was a consequence of toceranib therapy.

\section{CONCLUSIONS}

In this small cohort of cats which had advance disease, adverse events associated with treatment were mild (mainly grade I) and the majority were haematological or gastro-intestinal, which correlates with the main adverse events seen in dogs. Two of the eleven cats died from heart disease - it is unknown whether these cats had underlying heart disease at time of presentation as echocardiography was not performed. However, cardiotoxicity following treatment with sunitinib (the human analogue to toceranib) is well reported, and further investigation needs to be performed in veterinary patients.

\section{Survival following Oncept ${ }^{\circledR}$ melanoma vaccination in 30 dogs}

\section{Antonio Giuliano, Ola Marcinowska, Tess Oather, Malcolm Brearley, Jane Dobson}

Department of Veterinary Medicine, University of Cambridge, Cambridge, UK

\section{INTRODUCTION}

Oral melanoma is a common tumour in dogs, it carries a poor prognosis due to high metastatic rate. Digital melanoma in comparison are reported to behave slightly less aggressively. Melanoma vaccination with human tyrosinase DNA plasmid vaccine has been used for adjuvant treatment of oral and digit melanomas in dogs. Previous studies have reported conflicting outcomes, in terms of survival duration and in comparison to radiotherapy and / or surgery alone. The purpose of this study was to report our experience.

\section{MATERIAL AND METHODS}

Thirty dogs diagnosed with malignant melanoma treated with melanoma vaccine between the beginning of 2010 and the end of 2014 (23 oral, 4 digit, 1 pinna, 1 eyelid and 1 in the nose) were analysed retrospectively. All the dogs underwent complete staging and received a combination of surgery plus or minus radiotherapy and the melanoma vaccine.

\section{RESULTS}

Mean age of the 23 dogs with oral tumours was 9.8 years old with 14 male and 9 female, 7 Labrador, 4 cocker spaniel, 3 golden retriever, 3 flat coat retriever, 2 cross breed, 2 Irish setter, 1 Scottish terrier and 1 lurcher. Tumour sites were maxilla (6), lip (4), cheek (4), mandible (4), tongue (3) and not specified (2). 21 dogs completed the course of vaccination, 2 received only one injection. Ten dogs received at least 1 booster: one received 4 boosters, three 3 boosters, three 2 boosters, and three 1 booster. Mean survival for the 23 dogs was 424 days (range 133-1028). Sixteen dogs died of melanoma, with mean survival time of 369 days. Three dogs were still alive at the time of follow up (at 586, 626 and 954 days). Four dogs died of unrelated causes with a mean survival of 628 days (range 176-724). Fourteen patients were treated with surgery plus radiotherapy and thirteen surgery alone, average survival was 437 and 379 days respectively. Two patients were treated with radiotherapy alone and one did not receive any surgery or radiotherapy. For the digit melanoma patients mean survival was 287 days (range 90-714), eyelid 874, pinna 824 and the nose 133 days.

\section{CONCLUSION}

In this study the majority of patients died from metastatic disease and the total overall mean survival of 424 days suggests vaccination offers only a modest improvement in survival to that previously reported with surgery and or radiotherapy alone. 